\title{
Casein phosphopeptide in cow's milk is strongly allergenic
}

\author{
Teruaki Matsui ${ }^{1}$, Michihiro Naito ${ }^{2}$, Katsumasa Kitamura ${ }^{1}$, Atsushi Makino ${ }^{1}$, Yoshihiro \\ Takasato $^{1}$, Shiro Sugiura ${ }^{1}$, Hidehiko Izumi ${ }^{3}$, and Komei Ito ${ }^{1}$ \\ ${ }^{1}$ Aichi Children's Health and Medical Center \\ ${ }^{2}$ Nagoya University of Arts and Sciences \\ ${ }^{3}$ Nagoya University of Arts and Sciences School of Nutritional Science Graduate School of \\ Nutritional Science
}

September 24, 2021

\section{Conflict of interest disclosure}

The authors have no conflict of interest to declare.

\section{Financial support}

This study received no financial support.

\section{Keywords}

basophils, casein, phosphopeptide, food allergy, cow milk, milk hypersensitivity

To the Editor:

Cow's milk $(\mathrm{CM})$ is a common food allergen. ${ }^{1}$ It has a high casein content, which comprises approximately $80 \%$ of CM proteins. ${ }^{2}$ A variety of processed foods and medicines include processed products derived from $\mathrm{CM}$. Thus, attention must be paid to the symptoms induced by an allergic reaction to the accidental ingestion of these products. ${ }^{3}$

Casein phosphopeptide (CPP) is a processed product derived from caseins by tryptic digestion. ${ }^{4}$ It binds with amorphous calcium phosphate (ACP), increasing its ability to stabilize calcium phosphate in solution. ${ }^{5}$ Thus, CPP-ACP acts as a biologic calcium phosphate delivery vehicle and is used worldwide in various oral care products, such as chewing gums, topical creams, and toothpaste, owing to its anticariogenic properties. ${ }^{6}$

Because the allergenicity of CPP has yet to be determined, it is unclear whether CPP-ACP can induce allergic symptoms in patients with CM allergy (CMA). Thus, the purpose of this study was to validate the allergenicity of CPP-ACP. Our study was approved by the Research Ethics Board of Aichi Children's Hospital (approval number: 2020114) and conformed to the Declaration of Helsinki.

First, we searched the electronic medical record database of Aichi Children's Health and Medical Center from February 2012 to March 2021 to identify any CPP-ACP-related episodes. A total of eight patients were identified (Table 1). Data on the patients' characteristics, clinical information, episode details, and the associated results of the $\mathrm{CM}$ oral food challenge (OFC) test and blood tests were extracted from the medical records. The severity of the allergy-induced symptoms varied between patients. Although the amount of product used was small, of the six patients who reacted to the dental care product MI paste (GC Corp., Tokyo, Japan), which contains 10\% CPP-ACP, four developed anaphylaxis and required an intramuscular adrenaline injection. Two patients reacted to Recaldent gum (GC Corp.), which contains $0.18 \%$ CPP-ACP, and one of them experienced anaphylaxis. All patients had severe CMA and reacted to $<4 \mathrm{ml}$ of CM during the OFC. 
Next, we performed ImmunoCAP inhibition assays and basophil activation tests using CPP or casein to examine the in vitro allergenicity of CPP. We recruited those patients with CMA who were diagnosed by a positive CM-OFC test and were completely avoiding CM. Written informed consent was obtained from the parents of the participants at enrollment. Serum from patients $1 \mathrm{a}, 1 \mathrm{~b}$, and $1 \mathrm{c}$ and peripheral blood from patients $2 \mathrm{a}, 2 \mathrm{~b}$, and $2 \mathrm{c}$ were used for the inhibition assay and basophil activation tests, respectively (Table S1).

The casein, CPP, and ovalbumin (FUJIFILM Wako Chemicals, Osaka, Japan) used in this in vitro study were characterized by sodium dodecyl sulfate-polyacrylamide gel electrophoresis (Figure S1). All of the casein fractions (as1-, as2-, $\beta$-, and $\chi$-casein) were detected in the casein, and CPP showed a smear with a molecular weight $<20 \mathrm{kDa}$.

For the inhibition assay, each inhibitor was diluted with immunoglobulin (Ig) E Sample diluent (Thermo Fisher Diagnostics) and incubated at $4^{\circ} \mathrm{C}$ overnight with the target serum at a 1:1 ratio. Then, casein-specific IgE was measured by an ImmunoCAP test (Thermo Fisher Diagnostics, Tokyo, Japan). CPP inhibited caseinspecific IgE binding in a concentration-dependent manner in all three patients (Figure 1). Although the rate at which casein-specific IgE was inhibited was low at lower concentrations, it was $>80 \%$ when applied at the highest concentration $(10 \mathrm{mg} / \mathrm{ml})$. This inhibition was proved to be antigen-specific because $10 \mathrm{mg} / \mathrm{ml}$ of ovalbumin did not interfere with the detection of casein-specific IgE (Figure 1), and $10 \mathrm{mg} / \mathrm{ml}$ of casein and CPP did not inhibit egg white-specific IgE using the identical sera (Figure S2).

Next, the basophil activation test was performed using an Allergenicity Kit (Beckman Coulter Inc., USA), according to the manufacturer's instructions. Briefly, ethylenediamine tetraacetic acid (EDTA)-containing whole blood was incubated with various concentrations of casein, CPP, or ovalbumin for 15 min with a sufficient amount of calcium solution to override the chelating capacity of EDTA. Stimulations with $4 \mu \mathrm{g} / \mathrm{ml}$ anti$\mathrm{IgE}$ and phosphate-buffered saline were used for positive and negative controls, respectively. Phycoerythrin cyanine 7-conjugated anti-CD-3, fluorescein isothiocyanate-conjugated anti-CRTH2, and phycoerythrinconjugated anti-CD203c antibodies were added during the reaction. Using flow cytometry, the mononuclear cells were gated by forward and side scatter, and basophils were identified as CD3-CD294 (CRTH2) ${ }^{+}$cells. The percentage of activated basophils was determined using a threshold CD203c fluorescence intensity based on a comparison between the positive and negative controls (Figure S3). CPP activated basophils in a concentration-dependent manner comparable to that of casein in two of the patients (Figure 2a and 2b) but was less effective than casein in activating basophils at concentrations $>10 \mu \mathrm{g} / \mathrm{ml}$ in the remaining patient (Figure 2c).

These in vitro data clearly indicate that CPP possesses IgE-binding ability and induces basophil activation comparable to that of casein, which supports the fact that CPP causes severe allergic symptoms in patients with CMA. To date, many casein epitopes have been reported through IgE epitope mapping using synthetic peptides to predict CMA prognosis. ${ }^{7,8}$ However, only a few epitope sequences were located in the CPP regions, and no common epitopes were detected in the CPP regions of the different casein components. ${ }^{9}$

We previously reported a strong correlation between the IgE levels specific to $\alpha$ s1- and $\beta$-casein ${ }^{10}$ and confirmed strong cross-reactivity between them using competitive enzyme-linked immunosorbent assays. ${ }^{9}$ Although $\alpha$ s1- and $\beta$-casein showed a fairly low level of homology of total amino acid sequences (4\%-7\%), we found a partial similarity between the as1-casein (E61-K70) and $\beta$-casein (I12-E21) amino acid sequences, both of which were included in the CPP region. ${ }^{9}$ Based on these findings, we hypothesize the existence of common and dominant IgE epitopes in these regions.

Tryptic hydrolysis has been used to identify CPP in as1-casein (E61-K79), as2-casein (K1-Y20 and N46$\mathrm{K} 70$ ), and $\beta$-casein (R1-R25). ${ }^{4,9} \mathrm{CPP}$ contains highly phosphorylated serine residues in and around the core structure of the SSSEE motif. Therefore, the major phosphorylated epitopes in casein could have been missed in studies using non-phosphorylated synthetic peptides. ${ }^{9}$ Thus, phosphorylated serine residues in the $\mathrm{CPP}$ motifs could be critical in forming the dominant $\operatorname{IgE}$ epitopes in native casein components.

There are some limitations to our study. First, the inhibition assay revealed that CPP had a lower inhibitory 
ability than casein, but showed an almost identical basophil activating ability to that of casein. It was difficult to interpret the difference between the methods, which could be partially attributed to the small sample size. Nevertheless, the clinical fact that an anaphylactic reaction was induced by a small amount of CPP-ACP suggests strong allergenicity of CPP. Second, the CPP reagent used in our experiments was a mixture of trypsin-digested casein peptides, which might contain residual IgE epitopes, except for the CPP region in casein.

In conclusion, despite the fact that major epitopes of CPP have yet to be reported, CPP showed representative allergenic activity in casein. We are currently performing experiments using dephosphorylated CPP peptides and also planning to use synthetic phosphorylated CPP to confirm our study findings.

\section{Acknowledgments}

We thank all the physicians, nutritionists, and nurses who performed the OFCs and data collection, and Mr. Noboru Hayakawa (Clinical Laboratory technician in Aichi Children's Health and Medical Center) and Ms. Kumiko Matsumoto (Cell Biology Section, BML, Inc.) for their excellent technical assistance.

\section{Impact statement}

CPP could cause severe allergic reactions in patients with CMA. Moreover, it possesses IgE-binding ability and induces basophil activation comparable to that of casein

\section{Data availability statement}

The datasets of this study are not publicly available to protect the participants' personal data but can be availed from the corresponding author on reasonable request.

\section{References}

1. Peters RL, Krawiec M, Koplin JJ, Santos AF. Update on food allergy. Pediatr Allergy Immunol 2021; 32: $647-657$.

2. Ito K, Futamura M, Moverare R, et al. The usefulness of casein-specific IgE and IgG4 antibodies in cow's milk allergic children. Clin Mol Allergy 2012; 10: 1.

3. Ebisawa M, Ito K, Fujisawa T, Committee for Japanese Pediatric Guideline for Food Allergy TJSoPA, Clinical I, Japanese Society of A. Japanese guidelines for food allergy 2020. Allergol Int 2020; 69: 370-386.

4. Cruz-Huerta E, Garcia-Nebot MJ, Miralles B, Recio I, Amigo L. Caseinophosphopeptides released after tryptic hydrolysis versus simulated gastrointestinal digestion of a casein-derived by-product. Food Chem 2015; 168: 648-655.

5. Azarpazhooh A, Limeback H. Clinical efficacy of casein derivatives: a systematic review of the literature. J Am Dent Assoc 2008; 139: 915-924.

6. Bijle MNA, Yiu CKY, Ekambaram M. Calcium-based caries preventive agents: a meta-evaluation of systematic reviews and meta-analysis. J Evid Based Dent Pract 2018; 18: 203-217.

7. Caubet JC, Lin J, Ahrens B, et al. Natural tolerance development in cow's milk allergic children: IgE and IgG4 epitope binding. Allergy 2017; 72: 1677-1685.

8. Savilahti EM, Kuitunen M, Valori M, et al. Use of IgE and IgG4 epitope binding to predict the outcome of oral immunotherapy in cow's milk allergy. Pediatr Allergy Immunol 2014; 25: 227-235.

9. Naito M, Matsui T, Yamada C, Tagami K, Ito K, Izumi H. Evaluation of cross-reactivity between casein components using inhibition assay and in silico analysis. Pediatr Allergy Immunol 2021; 32: 544-551.

10. Naito M, Matsui T, Tagami K, et al. Changes in casein component-specific antibody levels during oral immunotherapy for milk allergy. Pediatr Allergy Immunol 2020; 31: 421-424.

\section{Tables}


TABLE 1 Allergic events caused by casein phosphopeptide-amorphous calcium phosphate

\section{Figure legends}

FIGURE 1 Inhibition assay of casein-specific IgE using ImmunoCAP tests.

Different concentrations of casein (black circles), casein phosphopeptide (white circles), or ovalbumin (gray circle) were mixed with the target serum at a 1:1 ratio and then incubated at $4^{\circ} \mathrm{C}$ overnight. Casein-specific IgE was measured using ImmunoCAP tests.

FIGURE 2 Basophil activation test stimulated by casein or casein phosphopeptide.

The basophil activation test was performed using an Allergenicity Kit (Beckman Coulter). Different concentrations of casein (black circles) and casein phosphopeptide (white circles) were used as stimulators. The percentage of activated basophils was determined using a threshold CD203c fluorescence intensity based on a comparison between the positive and negative controls.

\section{Hosted file}

Table.pptx available at https://authorea.com/users/336798/articles/538542-caseinphosphopeptide-in-cow-s-milk-is-strongly-allergenic

\section{Hosted file}

Fig_1.pptx available at https://authorea.com/users/336798/articles/538542-caseinphosphopeptide-in-cow-s-milk-is-strongly-allergenic

\section{Hosted file}

Fig_2.pptx available at https://authorea.com/users/336798/articles/538542-caseinphosphopeptide-in-cow-s-milk-is-strongly-allergenic 